\title{
Précarité professionnelle dans les emplois d'éducateur sportif à temps partiel
}

\author{
Professional precarity in part time sports coach jobs
}

\section{Eric Boutroy}

Maître de conférences, Sociologie, L-ViS (EA 7428) Université Lyon 1 - UFR STAPS, Université Lyon 1, 27-29 boulevard du 11 Novembre 1918, 69622 Villeurbanne cedex, France

Cécile Collinet

Professeure des universités, Sociologie, ACP (EA 3350) Université Paris-Est Marne la Vallée

- Université Paris-Est Marne-la-Vallée, 5 boulevard Descartes, 77454 Champs-sur-Marne, France

\section{Guillaume Routier}

Maître de conférences, Sociologie, L-ViS (EA 7428) Université Lyon 1 - UFR STAPS, Université Lyon 1, 27-29 boulevard du 11 Novembre 1918, 69622 Villeurbanne cedex, France

\section{Pour citer cet article :}

Boutroy, E., Collinet, C., \& Routier, G. (2020). Précarité professionnelle dans les emplois d'éducateur sportif à temps partiel. Sociologie, 11(1), 23-37.

Disponible à l'adresse suivante : https://journals.openedition.org/sociologie/6420 


\title{
Résumé
}

Les éducateurs sportifs caractérisés par une activité centrée sur le face-à-face pédagogique représentent la majorité des salariés de la branche du sport. Si leur nombre ne cesse de croitre laissant supposer un fort dynamisme de ce secteur d'emplois, la qualité de ces derniers interpelle du fait du poids considérable du temps partiel. Comment comprendre l'importance des faibles quotités ? Quels en sont les impacts dans les parcours et les perspectives professionnels ? À partir d'une enquête qualitative par entretiens auprès de salariés en situation d'emploi à temps partiel $(n=44)$ et d'employeurs $(n=29)$, il est possible de montrer que derrière des logiques d'emplois secondaires choisis, la plupart des situations relèvent du sous-emploi. Entre précarité d'intégration et d'exclusion, fragilité et satisfaction, les trajectoires professionnelles mettent en évidence un secteur sportif ambivalent fondée sur l'« entre-deux ».

\section{Mots-clés}

Éducateurs sportifs, Emploi, Précarité, Temps partiel, Trajectoires professionnelles

\begin{abstract}
Sports coach, characterized by face-to-face instruction, represent the majority of salaried employees in the sports sector. While their number continues to grow, suggesting a strong dynamism in this sector of employment, the quality of these jobs is a matter of concern because of the considerable weight of part-time work. How is it possible to understand this phenomenon? What are the impacts on career paths and professional expectations? Based on a qualitative interview survey of employees in part-time salaried employees $(n=44)$ and employers $(n=29)$, it is possible to show that behind selected secondary employment logics, most situations are underemployment. Between precariousness of integration and exclusion, fragility and satisfaction, professional trajectories highlight an ambivalent professional sector based on "in between" jobs.
\end{abstract}

\section{Keywords}

Employment, Part time job, Precariousness, Professional trajectories, Sports coach 


\section{Introduction}

L'emploi sportif a fait l'objet de nombreuses recherches et rapports, en particulier depuis le début des années 2000 (Falcoz \& Walter, 2009). En dépit de difficultés méthodologiques bien renseignées ${ }^{1}$ (Falcoz, 2013), les travaux centrés sur l'objectivation quantitative des emplois du secteur (Le Roux, 2006) ont mis évidence un important volume d'emplois et une indéniable croissance salariale depuis les années 1980. Selon un rapport récent (GAREF, 2014), l'emploi dans le secteur du sport (au sens des nomenclatures officielles) compte environ 350000 salariés et la branche professionnelle du sport au sens strict en compte 125 600. Par ailleurs, si les associations sportives sont des structures essentiellement bénévoles, l'emploi y a progressé de plus de $56 \%$ entre 1990 et 2000 (Falcoz \& Walter, 2007). Ces travaux et expertises ${ }^{2}$, parfois engagés dans le cadre de la mise en place du dialogue social dans le secteur du sport $^{3}$, ont contribué à faire émerger et s'établir l'idée d'un marché spécifique, « régulièrement précaire, et encore faiblement régulé » (Fleuriel, 2016, p. 13). Issu d'une étude commanditée par la branche sport ${ }^{4}$, cet article se concentre sur un des types d'emploi caractéristique du marché sportif : celui des éducateurs sportifs salariés (relevant de la CCNS). Même si la définition du métier et de ses compétences est discutée (Bernardeau-Moreau \& Collinet, 2009), il s'agit avant tout d'emplois de service relevant d'activités d'animation et d'encadrement du sport (requérant au sens strict des compétences sportives mais également des compétences éducatives ou de prise en charge de publics à besoins spécifiques). Ils représentent la plus grosse part des salariés de la branche sport et seraient en constante expansion (Quadrat-Études, 2018). D'après la Nomenclature d'Activités Française (NAF rév. 2 code 424-A), ils représentaient, en 2014, 65\% des salariés affiliés à la CCNS. Cependant, la progression du salariat dans le secteur sportif peut être

\footnotetext{
${ }^{1}$ Sans même parler des limites bien connues de la statistique publique (Le Roux \& Aguettant, 2006), la définition même de ce qu'est un emploi ou un établissement sportif influe considérablement sur le périmètre et les caractéristiques de ce marché.

${ }^{2}$ Notamment les travaux du RUNOPES (Le Roux, 2002), ou de l'OMNAS (2006) ainsi que du CAFEMAS (Pierre \& Collinet, 2014) résultant pour ces deux derniers de l'exploitation des chiffres des DADS (déclaration annuelle de données sociales de 2010) retraités par la DARES.

${ }^{3}$ Ils ont notamment abouti à la récente Convention Collective Nationale du Sport (CCNS) en 2005, dont la mise en place « par le haut » a été bien décrite par Fleuriel (2013).

${ }^{4}$ Ce travail est issu d'un projet de recherche réalisé dans le cadre d'une convention avec la branche professionnelle sport (regroupement des structures relevant de la CCNS) qui souhaitait mieux connaître les profils et parcours des salariés à temps (très) partiel).
} 
relativisée : $80 \%$ des associations sportives n'ont pas de salariés et $80 \%$ des clubs employeurs ont moins de 3 salariés (Malet \& Bazin, 2014). Surtout, la faible quotité horaire est une caractéristique de ce secteur puisque $42 \%$ des éducateurs sportifs (toutes branches confondues) travaillent à temps partiel (Devetter \& Fleuriel, 2016) et que $40 \%$ des salariés relevant de la CCNS sont en situation d'emploi annexe ${ }^{5}$ (Gouju, 2014). Partant de tels constats, notre étude a ciblé et questionné les situations de faible temps de travail. Comment comprendre un tel poids des faibles quotités dans la branche sport? Dans quelle mesure est-il en lien avec des parcours de salariés marqués par des situations de sous-emploi et de précarité professionnelle ? Ces salariés se considèrent-ils euxmêmes comme des «exclus »?

La notion de précarité a une histoire complexe (Paugam, 2000 ; Tasset, 2017). La banalisation de son usage en France dans le sens commun va de pair avec une prolifération des définitions sociologiques et une extension plus ou moins grande de son périmètre (Barbier, 2005 ; Bresson, 2007) pouvant aboutir à un questionnement de sa pertinence (Vultur, 2010). Dans une approche compréhensive, l'usage de cette « catégorie ordinaire de la compréhension » (Bresson, 2011) permet de comprendre les représentations et vécus des individus au regard d'une «norme d'emploi » encore marquante (Ibid. ; Eckert, 2010) : les emplois à temps plein stables (ou typiques) et les protections qui y sont associées. Dans ce sens courant et plutôt extensif, la précarité est ici appréhendée comme une expérience professionnelle d'instabilité, de discontinuité ou encore d'insécurité, imposée ou «choisie » sous contrainte (Ghesquière, 2014). Nous sommes ici attentifs à l'instabilité, c'est-à-dire aux aléas qui affectent le revenu d'une personne (Villeneuve, 1984) tout autant qu'à la fragilité, entendue comme «exposition à des risques de différentes natures et une faible capacité à $[\mathrm{y}]$ faire face, imputable à un défaut de ressources de divers ordres » (De Peretti, 2005). Elle peut être liée à un certain nombre de critères objectivables, relevant d'abord de la précarité de l'emploi au sens de Paugam (2000). Un premier aspect qui caractérise la population étudiée dans ce travail est le possible temps partiel imposé. Cette précarité de l'emploi peut se combiner avec des contrats à «statut précaire ( $\mathrm{CDD}$, intérim, etc.) ou des emplois stables déstabilisés produisant « une forte vulnérabilité économique et une restriction, au moins potentielle,

\footnotetext{
${ }^{5}$ L'emploi annexe est défini comme un emploi représentant moins de $120 \mathrm{~h}$ ou 30 jours cumulés par an.
} 
des droits sociaux » (Ibid., p. 356). Mais nous nous intéressons également à la précarité du travail (Ibid.), lorsque les conditions de travail sont dégradées ou insatisfaisantes et qui impliquent d'autres indicateurs : rétribution insuffisante, faible reconnaissance par l'employeur, inintérêt du travail voire carence d'appartenance à un collectif. Suivant Béroud et Bouffartigue (2009), nous avons choisi de regrouper ces éléments sous la notion englobante de précarité professionnelle qui permet de prendre en compte la pluralité des dimensions du rapport à l'emploi et au travail (Malenfant, LaRue \& Vézina, 2007). La précarité laisse donc entrevoir des parcours discontinus associés à l'emploi et aux expériences qui en résultent (Cingolani, 2005). Ils se rencontrent dans d'autres secteurs qui, à l'instar de l'animation ou du service à la personne par exemple, présentent certaines caractéristiques similaires au premier regard: multiplicité d'employeurs peu professionnalisés, importance du temps partiel, flexibilité de la main d'œuvre, recours aux contrats de travail précaires (CDD, contrats aidées), etc. (Lebon \& Lescure, 2006 ; Angeloff, 2008) ${ }^{6}$.

Notre étude, ciblant des salariés en possible situation de sous-emploi, s'efforce d'appréhender non seulement comment le temps partiel peut se cumuler avec d'autres indicateurs de précarité professionnelle, mais également de quelle manière ces critères peuvent renvoyer ou non à un vécu de fragilité ou d'exclusion. S'intéresser à l'expérience et aux trajectoires permet en effet d'accéder à la dimension subjective de la précarité : inquiétude et sentiment de vulnérabilité en lien avec cette instabilité et/ou incertitude. Car on ne peut se limiter à la question de la quotité ou du statut pour comprendre «l'insécurisation des trajectoires professionnelles des individus » (Bouffartigue, 2011, p. 3). Le sentiment de précarité est ainsi susceptible de variations (entre individus, entre secteurs professionnels), et de ne pas être nécessairement liée aux indicateurs objectifs de précarité (Fournier, Bourassa \& Béli, 2003). Dans cette perspective, les caractéristiques de l'emploi doivent être articulées à celles des parcours (professionnels et biographiques) pour rendre compte du vécu de précarité. Il s'agit de prendre en compte chez les salariés de la branche sport « l'ensemble de la vie de travail avec les emplois exercés et les phases de transition qui constituent des écarts au point de référence défini comme un travail stable à temps plein pour un salaire fixe »(Ortiz, 2009, p. 268). Ainsi, la notion de

\footnotetext{
${ }^{6}$ Sans que ce soit un axe central de l'article, nous nous permettrons quelques références à ces secteurs à la fois proches et distants pour mieux identifier de possibles particularités de la branche sport.
} 
trajectoire est complémentaire de celle de la situation d'emploi en incluant des dimensions conflictuelles inhérentes aux parcours; notamment la dimension potentiellement subie, ou choisie sous contraintes, des situations de faible quotité. Ce point est essentiel car le temps de travail à temps partiel renvoie à deux logiques différentes. D'un côté, celle du travail à temps réduit par « un aménagement individuel et volontaire du temps de travail » (Ulrich, 2009, p. 210). D'un autre côté, celle du sousemploi, « qui résulte d'une embauche à temps partiel décidée par l'employeur » (Id.) qui se caractérise par une disponibilité à travailler plus et/ou à rechercher un autre emploi. Le temps partiel subi doit lui-même être replacé dans une conception «dynamique » selon qu'il renvoie à une «précarité d'intégration» ou d'《exclusion» (Nicole-Drancourt, 1992). D'autant plus que le sport est un secteur professionnel marqué par l'engagement passionnel (Chimot \& Schotté, 2006), on peut alors se demander si des situations professionnelles atypiques peuvent être associées à des expériences subjectives positives et des perspectives satisfaisantes (Vultur, 2010). En ce sens, l'article va souligner en quoi la précarité dans la branche sport pourrait parfois relever moins d'une exclusion que d'une « intégration incertaine » (Paugam, 2000), symptomatique d'un « entre-deux » (Bresson, 2011) entre « centre » de la société (noyau dur d'un salariat typique) et « périphérie » (des précaires marginalisés et exclus). Comprendre de tels phénomènes implique une enquête de terrain à deux entrées.

\section{Encadré méthodologique}

Inscrit dans une approche qualitative de la précarité professionnelle, ce travail donne la parole aux travailleurs étant, ou ayant été, en situation de temps partiel dans la branche sport afin de confronter la caractérisation des parcours professionnels avec l'expérience vécue par l'individu. Il s'agit en cela de documenter et analyser les possibles expériences de précarité vécues par ces salariés de la branche sport. 44 entretiens semi-directifs ont été réalisés avec des salariés afin de décrire leurs situations professionnelles actuelles et reconstituer leurs carrières ${ }^{7}$. Tous les salariés interrogés ont un emploi comportant une

\footnotetext{
${ }^{7}$ La grille d'entretien portait sur la caractérisation du parcours scolaire et sportif, des situations d'emploi actuelles et passées (qualité d'emploi, conditions de travail) et des perspectives professionnelles. Elle s'intéressait aussi au vécu de cette trajectoire professionnelle en termes d'instabilité ou non.
} 
dimension d'enseignement (liste en annexe 1) ; certains sont en emploi principal ${ }^{8}$ dans la branche (32), d'autres en emplois secondaires (12). Même si ce sont les premiers qui nous intéressent le plus, les seconds présentent l'intérêt de témoigner par leurs trajectoires soit d'une potentielle période préalable d'emploi principal dans la branche, soit d'une volonté contrariée d'entrer dans la branche en emploi principal. L'échantillon construit n'a pas vocation à être représentatif de la population étudiée car il a été centré sur des salariés travaillant, ou ayant travaillé, à temps (parfois très) partiel dans la branche sport. À partir de ce trait commun, nous avons cherché à construire une série de cas diversifiés. Les salariés interviewés ont été choisis en veillant, notamment, à une diversification des âges (de 22 à 55 ans), des activités sportives (et donc des sous-secteurs professionnels afférents $^{9}$ ), des temps de travail (temps plein ou partiel, voire emploi annexe au moment de l'entretien). Les interviewés salariés sont cités dans l'article de manière anonyme (S.1, S.2, etc.). 29 entretiens d'employeurs ont également été réalisés à titre secondaire afin de prendre en compte les enjeux de l'emploi au regard de leurs problématiques spécifiques. Réalisés avec des dirigeants (liste en annexe 2) à propos de leurs salariés, ils abordent les questions de leurs difficultés en tant qu'employeur, de la possible imposition de l'emploi partiel pour des raisons de flexibilité, de leur politique salariale comme de leur plan de développement. Les employeurs ont été choisis selon le même principe de diversification soumis aux entretiens salariés (type de structure, taille, activités sportives concernées). Les interviewés employeurs sont cités sous un format comparable à celui des salariés (E.1, E.2, etc.).

Dans un registre plutôt explicatif, nous commencerons par voir quelles sont les logiques à l'œuvre derrière le recours important à l'emploi à temps partiel dans la branche sport. Il s'agira de démêler les raisons entre choix personnel des salariés et gestion flexible de la main d'œuvre par l'employeur, mais aussi de montrer comment la pluralité des situations de temps partiel (choisies ou subies) s'accumule avec d'autres caractéristiques

\footnotetext{
${ }^{8}$ Un emploi est dit principal dès lors qu'il est actif (il donne lieu à une rémunération) et non annexe. En conséquence, il s'agit généralement de l'emploi le plus rémunérateur pour l'individu. Il peut donc être à temps partiel.

${ }^{9}$ Cette diversité, liée à la nature de la demande initiale du commanditaire de l'étude, constitue une limite du travail. En effet, le marché de l'encadrement sportif recouvre des sous-secteurs assez différents en termes de degré de professionnalisation, de capacité à rémunérer le travail, etc. Cependant, s'il n'a pas prétention à pouvoir être généralisé, ce panorama général visait à identifier des possibles traits partagés permettant de constater un air de famille entre les situations décrites, avant de possibles enquêtes ultérieures plus resserrées.
} 
de la précarité professionnelle à la fois dans l'emploi et le travail. Nous verrons ensuite au travers de l'expérience vécue des travailleurs de quelle manière le temps partiel s'intègre dans des dynamiques oscillant entre précarité d'intégration et précarité d'exclusion faisant peut-être de la branche un secteur professionnel caractéristique des « situations sociales de l'“"entre-deux"” décrites par Bresson (2011).

\section{Logiques du temps partiel et formes de précarité chez les éducateurs sportifs}

La nature même du métier d'éducateur sportif (relation de service durant les temps libres des bénéficiaires) explique que l'emploi se fasse souvent à temps partiel, avec des horaires flexibles et décalés (Loirand, 2006 ; Falcoz, 2016). Cet émiettement et cette flexibilisation du temps de travail sont des contraintes structurelles pour l'emploi d'éducateur sportif. Beaucoup d'établissements offrent en effet, chacun, relativement peu d'heures de face-à-face, qui plus est sur des horaires similaires (fin de journée, mercredi, week-end) :

Certains cours se déroulent à la même heure (débutants / confirmés). Les adhérents sont tous libres aux mêmes heures, c'est-à-dire après le travail, il est donc plus facile de multiplier les enseignants sur les mêmes créneaux que les soirs de semaine (E.5, gérante d'une salle privée d'arts martiaux).

Les clubs de notre échantillon n'étaient ainsi en capacité d'offrir que 2 à $20 \mathrm{~h}$ de travail par semaine à un même éducateur. Ce dernier se trouve alors dans ce qui peut être une contrainte double : travailler à temps partiel pour un employeur avec l'impossibilité de travailler sur le même créneau pour un autre. Parfois l'éducateur manque de compétences ou d'envie pour prendre en charge la variété des publics qui composerait une quotité satisfaisante :

Par ailleurs, pour n'avoir qu'une seule personne (à temps plein) il faudrait qu'elle accepte d'enseigner à tous les publics (enfants, adultes, débutants, confirmés) or, les professionnels qui travaillent chez nous sont des intervenants gradés d'un très haut niveau technique. Ils refusent souvent d'enseigner aux enfants, voire ne savent pas le faire (idem).

Une autre forme de «partiellisation » du temps de travail est liée à la saisonnalité de certains marchés, notamment les activités de plein air touristiques. La demande en 
personnel subit les périodes d'affluence des vacances et ne peut se penser que sur des durées intenses et limitées (pic d'activité en pleine saison, creux ensuite).

Ils [Les éducateurs] travaillent sept mois, grosso modo, plutôt quatre mois l'hiver, deux mois et demi/trois mois l'été, c'est à peu près ça. Et ensuite ils sont souvent au chômage. Parce que de toutes façons travailler chez d'autres employeurs ça serait travailler plutôt à l'étranger par rapport aux saisons et donc il n'y en a quand même pas beaucoup qui le font. (...) En surf par exemple, ils tiennent tous à faire quatre mois de saison parce qu'au bout de quatre mois ils déclenchent leur droit à pôle emploi donc la plupart soit ils vont travailler à l'étranger l'hiver, soit ils sont au chômage ou ils font d'autres petits boulots (E.9, référent national VTT UCPA).

Ce témoignage montre l'ambivalence du temps de travail partiel : parfois choisi (comme faire de la saisonnalité un mode de vie), d'autres fois subi (par exemple alterner petits emplois et inactivité).

\section{Temps partiel choisi et emploi secondaire}

Plusieurs interviewés témoignent d'une situation professionnelle satisfaisante, où un emploi d'éducateur à faible quotité choisie (entraîneur de rugby, tennis ou roller ou éducatrice polyvalente) est un complément d'activité qui s'ajoute à un emploi à temps plein dans le sport (professeurs d'EPS, ETAPS ${ }^{10}$, Professeur dans l'armée : S.4, S.9, S.30, S.37, S.38) ou dans un autre secteur d'activité (employée de mairie, infirmière : S.24, S.34). Il s'agit à chaque fois d'une activité permettant une rémunération additionnelle, mais aussi de garder un lien avec une activité «plaisir » ou « passion », avec la stabilité d'un emploi principal ailleurs (CDI, titulaire de la fonction publique).

S.37 (femme, 43 ans), professeure d'EPS et maître-nageur en emploi annexe illustre ce lien fort avec une activité exercée pour le plaisir. Son parcours est assez linéaire : étude en STAPS ${ }^{11}$, passage du BNSSA ${ }^{12}$, puis réussite du CAPEPS ${ }^{13}$. Rapidement stabilisée en tant qu'enseignante d'EPS titulaire, elle continue à avoir des emplois annexes dans la branche sport qui ont rempli des fonctions différentes selon les périodes de sa vie.

\footnotetext{
${ }^{10}$ Éducation Physique et Sportive, Éducateur Territorial des Activités Physiques et Sportives

${ }^{11}$ Sciences et techniques des Activités Physiques et Sportives

${ }^{12}$ Brevet National de Sécurité et de Sauvetage Aquatique

${ }^{13}$ Sciences et Techniques des Activités Physiques et Sportives, Brevet National de Sécurité et de Sauvetage Aquatique, Certificat d'Aptitude au Professorat d'Éducation Physique et Sportive.
} 
Au départ sur cet emploi, j'étais étudiante donc j'avais besoin de travailler. (...) C'était une histoire financière mais je n'ai jamais arrêté ce truc-là. Je pense que c'est que j'aime ça. Je n'aime pas être le soir devant la télé. Très sincèrement, n'être que prof d'EPS avec des gamins difficiles et rebondir dans une association avec la sécurité, c'est agréable. Bon j'étais crevée le soir... Mais si je m'interroge sincèrement $\mathrm{j}$ 'aimais faire ça quand même.

Ces emplois secondaires choisis lui permettent de compenser et s'épanouir hors d'une institution parfois pesante :

Moi ce que j'aime, c'est la diversité dans mon travail. Ça représente 20h de travailler dans un bahut avec des enfants difficiles, et moi dans les associations je rencontre des gens différents, des professions libérales. (...) Je crois que je suis faite pour travailler et avoir différentes vies professionnelles.

Ces situations sont satisfaisantes pour les interviewés, mais il peut demeurer une volonté de faire carrière dans la branche sport en y augmentant son temps de travail. C'est le cas pour S.9 (homme, 50 ans), prêt à une bifurcation professionnelle malgré l'identification des limites du secteur (charge de travail, rémunération). Professeur agrégé d'EPS, il a été joueur professionnel puis entraîneur dans de nombreux clubs de rugby jusqu'en D1 :

Si demain on me proposait le poste de responsable du centre de formation du S., si je me mets dans la peau du responsable sportif qui ne fait que ça, oui je prendrais le poste parce que ça peut être un tremplin vers d'autres postes. Mais je trouve le salaire, par rapport à la charge de travail et par rapport à un club qui a 25 millions d'euros de budget, pas du tout adapté. À mon avis il est deux fois inférieur à ce qu'il devrait être.

S.34 (femme, 31 ans) est infirmière à temps plein et a un emploi annexe d'éducatrice de tennis pour une répartition de temps de travail évaluée à $80 \% / 20 \%$ :

À long terme si j'étais sur un 50/50 ce serait top. Sachant que dans le 50/50 ce que j'aimerais c'est avoir un peu des activités annexes. Je ne pense pas [faire] que du terrain : faire un peu de formation, de préparation physique. Pas forcément avec une raquette dans la main, ça pourrait être de l'accompagnement de stagiaire, de la préparation physique.

La logique du temps partiel ici est clairement celle d'un « aménagement individuel et volontaire du temps de travail» (Ulrich, 2009, p. 210) par des choix progressifs et réversibles. 
Si tous les employeurs de club interviewés soulignent pour eux l'intérêt de l'emploi à faible amplitude du fait des contraintes d'activité (horaires restreints et publics variés), ils le mettent en perspective avec cette conception spécifique du métier d'éducateur sportif. Il s'agirait d'un emploi par nature secondaire, l'illustrant par la figure de l'enseignant d'EPS qui, le mercredi après-midi ou le soir en semaine, s'investit dans un club pour encadrer les jeunes. De la sorte, le travail d'éducateur sportif est envisagé comme emploi d'appoint visant un complément de salaire pour des personnes ayant un emploi principal ailleurs. Il s'agit aussi parfois d'avoir un « job » pour des étudiants :

Les entraîneurs qui font la gym le soir, ils font leurs deux ou trois heures et ils ont un autre boulot, ou ils sont étudiants. Il en faut des petits boulots. C'est pratique. Cela permet à chacun de s'exprimer en fonction de leur emploi du temps... Si on a des études à côté, cela permet de faire un petit boulot à côté (E.3, président d'un club de gymnastique sportive).

Cette focalisation régulière des employeurs interviewés sur la figure idéale de l'emploi secondaire choisi peut être interprétée comme un moyen d'évacuer la problématique du temps partiel subi. Cette figure idéale de l'emploi secondaire peut être rapprochée des « occasionnels » de l'animation (Lebon, 2010). En effet, les détenteurs des diplômes de l'animation comme le BAFA ou le $\mathrm{BAFD}^{14}$ exercent majoritairement à titre occasionnel ; souvent lycéens et étudiants, l'animation est pour eux un « petit boulot » (Camus, 2008). Très peu se dirigent vers une carrière dans l'animation et la gestion de ce personnel occasionnel se révèle du coup moins coûteuse en termes de gestion des ressources humaines pour l'employeur. Ainsi, dans le secteur du sport, la structuration du marché de l'emploi proposée par les employeurs enferme potentiellement le salarié dans un temps partiel contraint ne lui permettant pas d'envisager une carrière professionnelle en tant qu'éducateur sportif, même si celle-ci est envisagée et/ou souhaitée. Cette appréhension de l'emploi secondaire et sa structuration ne contraignent pas non plus l'employeur à penser le développement de l'emploi dans sa structure pour proposer une augmentation des volumes horaires permettant de stabiliser le salarié dans un emploi principal.

\footnotetext{
${ }^{14}$ Brevet d'Aptitude aux Fonctions d'Animateur de centre de vacances et de loisir et Brevet d'Aptitude aux Fonctions de Directeur d'accueils collectifs de mineurs.
} 


\section{Temps partiel subi et précarité}

L'embauche à temps partiel est avant tout décidée par l'employeur dans une logique de gestion flexible de la main d'œuvre pour répondre à ses contraintes : durée de travail courte, besoins de compétences multiples, horaires contraignants et atypiques, périodes de travail irrégulières, etc.

Nous, on a fait le pari d'embaucher les personnes à travers les réseaux et qu'ils fassent eux-mêmes leurs temps complets (...). La personne va se faire un ETP [emploi à temps plein] avec une polyvalence sportive sur différents publics et sur différents secteurs. Ce n'est pas possible autrement pour un ETP (E.2, Président d'un comité départemental du Sport en milieu rural)

Ce témoignage d'employeur est explicite : c'est d'abord au travailleur de trouver des ressources (réticularité et emploi de gré à gré, multicompétences en lien avec la réglementation par les diplômes, cumul d'emplois) pour faire face à ces volumes insuffisants proposés par les structures. Cette forme assez poussée d'individualisation de la relation salariale n'est pas caractéristique de la branche sport : elle est par exemple centrale dans le secteur des services à la personne (Ribault, 2008).

La capacité à cumuler des emplois multiples d'éducateur est donc une manière dont 15 interviewés précisent aujourd'hui compenser les faibles quotités ${ }^{15}$. S.5 (femme, 48 ans) est éducatrice sportif à la fédération d'Éducation Physique et Gymnastique Volontaire (EPGV). Titulaire d'un Brevet d'État (BE) de gym, elle a multiplié les employeurs et les emplois (jusqu'à huit structures), a diversifié ses diplômes (formation fédérale pour intervenir sur une spécialité «sport-santé ») et est même devenue tutrice et jury pour le passage des $\mathrm{CQP}^{16}$.

En cumulant toutes les associations et l'école des sports ça me fait environ 1800 euros par mois. Depuis 1997, j'ai multiplié et ça y est : pour en vivre correctement, il faut au moins 23 heures de cours par semaine. (...) [La situation est] satisfaisante, mais pas stable. Parce qu'une association, on a une petite épée de Damoclès au-dessus de la tête, c'est des contrats qui sont reconduits chaque mois de septembre, avec un bilan de ce qu'on a fait pendant l'année. Et puis il peut arriver qu'un président parte d'une association, et une

\footnotetext{
${ }^{15} \mathrm{Si}$ l'on prend en compte le parcours professionnel, la quasi-totalité de notre échantillon a vécu au moins une période de multiactivité.

${ }^{16}$ Certificat de qualification professionnelle : diplôme délivré par la branche professionnelle et permettant d'exercer contre rémunération mais avec des contraintes liées au nombre d'heures réalisées et à la population encadrée.
} 
association ne peut vivre que s'il y a un président, un trésorier, un secrétaire, donc voilà. Un jour ça peut arriver qu'une association n'ait plus de président et, à ce moment-là, je suis au chômage. Ma situation me convient, moi je fonctionne comme ça, donc quand je me sens bien je reste et puis voilà. (...) J'ai la chance d'être sur plusieurs associations donc on va dire que je ne mets pas mes œufs dans le même panier, c'est ce qui m'assure un peu plus de stabilité que certains.

Elle témoigne d'une situation ambiguë : constat d'une forme de satisfaction et confiance dans la possibilité de travailler (en se comparant à d'autres hypothétiques éducateurs moins stabilisés) mais sentiment d'insécurité et de fragilité latente.

La faible quotité subie et la multiplication des employeurs s'additionnent également à d'autres indicateurs de précarité de l'emploi, en particulier le recrutement sous statut précaire: CDD de durée variable ${ }^{17}$, vacations voire auto-entreprenariat ${ }^{18}$. Cette diversification des statuts peut être imposée par l'employeur ou être choisie sous contrainte. C'est le cas en particulier de l'auto-entreprise (utilisée par 12 éducateurs interviewés). Elle permet d'assouplir les démarches administratives d'un recrutement par un club, d'optimiser à court terme les revenus au détriment des protections sociales et parfois de faciliter des activités complémentaires de coaching pour des particuliers. S.29 (homme, 39 ans) est en CDI à temps partiel dans un club d'athlétisme. Cette stabilité dans le sous-emploi le met en insécurité économique (rémunération de 500 euros par mois) et l'oblige à compléter son salaire par de l'auto-entrepreunariat en tant que coach individuel, puis à ajouter quelques heures d'encadrement en CDD dans un club basket-ball. Derrière l'autonomie et la liberté qu'elle promeut, cette sorte $\mathrm{d}^{\prime}$ « uberisation ${ }^{19} \mathrm{du}$ secteur sportif fragilise les protections sociales et favorise l'émiettement du travail. Contribuant à «un cumul de fragilités » (Abdelnour, 2017, p. 257), elle est donc source d'incertitude et de vulnérabilité pour les travailleurs (Deprost, Laffron \& Imbaud, 2013). Elle fragilise non seulement les dispositifs traditionnels de flexibilité (CDD, intérim par exemple) mais en

\footnotetext{
${ }^{17}$ Taux de CDD avoisinant les $40 \%$ en Ile de France par exemple (Source : L'insertion professionnelle des diplômés de niveau IV des secteurs du sport et de l'animation en Ile de France, 2014).

${ }^{18}$ L'auto-entreprenariat ou micro-entreprenariat a bien été identifié comme un outil pour essayer de sortir de la précarité en créant son emploi ou en répondant à des pressions d'employeurs, dans des situations de quasi-travail dissimulé (Giraud \& al., 2014). Dans la branche sport, le nombre d'indépendants a été multiplié par 2,5 en 8 ans (Quadrat, 2018).

${ }^{19}$ Ce terme récent et encore instable n'est pas référé ici à la plateformisation numérique des relations (Vétois \& Raimbault, 2017), mais au remplacement « du salariat, au profit d'interactions directes des travailleurs sans statut et des consommateurs » (Méda, 2017, p. 6).
} 
même temps les dispositifs donnant accès à des protections salariales pour les personnes ayant plusieurs employeurs (groupement d'employeurs ou portage salarial). Au regard de la similitude des tâches, il semble possible dans certains cas de parler de « salariés dissimulés » (Weber, 2008) ou «déguisés » (Abdelnour, 2017). Cette question de la requalification de la relation est d'ailleurs aujourd'hui au cœur des interrogations de nombre de fédérations sportives, notamment du fait que le recours à l'auto-entreprenariat crée ici rapidement des liens de subordination potentiels. Ainsi, comme le soulignent Botsman et Rogers (2011), si cette modification de la relation salariale peut parfois pour l'individu être une solution lui permettant de générer indépendamment un revenu, il ne s'agit en rien d'une solution miracle pour pallier les problématiques de l'emploi, en particulier dans le secteur associatif.

Cet effet déstabilisant est d'autant plus marqué que se multiplient aussi des dispositifs informels («borderline », selon les propres termes d'un Directeur Technique National [DTN] adjoint [E.24]), qui permettent d'échapper à la salarisation par le défraiement, l'utilisation du régime indemnitaire ${ }^{20}$, le recours aux gratifications de stage, le travail au noir voire le travail non rémunéré (par exemple des heures supplémentaires non payées) ou des gratifications matérielles ${ }^{21}$. Dans la majorité des entretiens réalisés avec des salariés, il ne semble pas y avoir d'alternative proposée par l'employeur. S.44 (32 ans), employé d'un comité départemental d'athlétisme, explicite ce fonctionnement courant :

\begin{abstract}
Au jour d'aujourd'hui dans l'athlétisme, il y a très très peu de personnes salariées en tant qu'entraîneur. Moi quand j'ai commencé, ça n'a pas été un métier, c'était juste des indemnités (...). Aujourd'hui, je combine donc le rôle d'entraîneur et de directeur de comité. Pour celui-ci je suis salarié, voilà. Au global, pour ce poste je touche environ 1400 euros net par mois. Et pour l'entraîneur, actuellement je suis indemnisé (...). Et là c'est une fourchette à peu près de 350/400 euros par mois.
\end{abstract}

\footnotetext{
${ }^{20}$ Le régime dit indemnitaire prend en compte toutes les primes et indemnités qu'un salarié peut toucher sans qu'elles ne rentrent directement dans le calcul de son salaire. Il s'agit, par exemple, des primes liées aux conditions de travail (sujétions, insalubrité, vêtements, astreintes, heures supplémentaires...) ou encore des indemnités de remboursement de frais (déplacement, missions, mobilité...).

${ }^{21} \mathrm{~S} .34$ (31 ans) infirmière et entraîneur de tennis a été «gratifiée » en matériel à ses débuts en emploi annexe décrit cette substitution du salaire en «biens » : «J'étais indemnisée mais ma première année, ça m'allait bien. Ma deuxième, j'ai fait beaucoup d'heures... Du côté des indemnités, à l'époque, on avait une somme d'argent à dépenser dans un magasin de sport, c'était dans le rayon tennis. Mais cette somme-là, pour moi, devenait compliquée. Je n'avais pas envie de dévaliser le rayon, je n'en avais absolument pas besoin ».
} 
Ces formes de contournement du salariat n'ont rien d'exceptionnel : 17 interviewés déclarent les avoir rencontrées au moins une fois dans leur parcours. On notera que certains «bricolages » peuvent être faits au bénéfice du salarié, telle cette personne titulaire du $\mathrm{BE}^{22}$ de Taekwondo qui a négocié un CDI soutenu par une aide (cofinancement). Officiellement à $35 \mathrm{~h}$ (mais sur un salaire horaire bas), l'emploi est à $24 \mathrm{~h}$ effectives, afin de lui permettre de compléter son salaire via des emplois annexes dans d'autres clubs grâce à d'autres spécialités (tir à l'arc, BNSSA). L'enjeu du volume horaire pour se rapprocher d'un emploi typique est d'autant plus important que le secteur sportif, en particulier associatif, se caractérise majoritairement par des niveaux de rémunération plus faible que la moyenne (Hely, 2008 ; Nirello \& Prouteau, 2016). Ainsi, beaucoup des salariés interviewés en temps partiel subi se disent insatisfaits de leur niveau de salaire, même si certains secteurs, comme celui du tennis (E.22, S.18), permettent parfois des niveaux de rémunération horaire considérés comme supérieurs aux autres secteurs, qui rendent des temps partiels à forte quotité (80\%) satisfaisants. Comme dans les services à la personne (Ribault, 2008), la précarité du travail est aussi liée à l'éparpillement des heures et des lieux de travail qui ne favorisent pas la stabilisation (fatigue, planning mouvant et complexe, nombreux déplacements non rémunérés).

Dans une logique de professionnalisation (Bernardeau-Moreau, 2004), certains employeurs voire structures fédérales témoignent d'engagements notables pour renforcer la stabilité des emplois. D'une part, il s'agit d'essayer d'augmenter les périodes d'activité en diversifiant les publics et les services : organisation de stages sportifs pour compenser le creux estival, développement des activités pour les seniors ou le (péri)scolaire en journée, etc.

Car pour avoir un ETP il faut faire des choses très variées : associatif, seniors... Il faut regrouper les choses. La personne va se faire un ETP avec une polyvalence sportive sur différents publics et sur différents secteurs. Ce n'est pas possible autrement pour un ETP (E.2, vice-président de la ligue de sport adapté).

La prise en charge de fonctions administratives ou managériales est une autre forme de polyvalence citée qui peut permettre d'augmenter le temps de travail. Mais ces possibilités exigent du travailleur de disposer de compétences et qualifications multiples

\footnotetext{
${ }^{22}$ Brevet d'État d'éducateur sportif.
} 
(ou de s'engager et être accompagné pour se former), et qu'il y trouve une reconnaissance. En ce sens ces tentatives de professionnalisation tendent à renforcer le transfert du processus de gestion des compétences vers l'individu. Elles encouragent la mobilisation des salariés, principaux responsables de leur employabilité, dans l'obtention des compétences nécessaires à l'atteinte d'un ETP. Notons toutefois que, lorsque les structures s'engagent et proposent d'accompagner leurs salariés dans cette voie du développement de leurs compétences, cette logique de polyvalence n'en reste pas moins difficile à faire accepter par tous. E.21 témoigne par exemple de salariés ayant une conception du métier d'entraîneur centrée sur la performance sportive qui les font se désintéresser à la fois d'autres publics moins légitimes (santé, loisir, etc.) et d'éventuelles missions ne relevant pas de l'entrainement au sens strict (tâches administratives notamment). Sur le marché de l'emploi et du travail sportif se rencontre donc une double problématique : celle de l'employeurabilité (Devetter \& Fleuriel, 2016) et celle de l'employabilité.

Les variations de quotité peuvent être liées paradoxalement à des dispositifs de stabilisation de l'emploi. Le recours au CDI Intermittent (CDII) permet par exemple de lisser la rémunération sur 12 mois (en cas d'inactivité estivale) : la stabilité du contrat ayant pour conséquence une baisse de quotité et de revenus. S.17 (homme, 40 ans), en CDII 25h/semaine dans un club de rugby, y voit une source d'insécurité économique et se considère de fait comme stable et précaire. A l'inverse, les dispositifs de mutualisation d'emplois (cités par 6 salariés) ou le groupement d'employeurs Profession Sport \& Loisirs $^{23}$ apparaissent comme un levier important pour favoriser le multi-emplois (prise en charge des tâches administratives, facilitation de l'appariement et du recrutement) et renforcer la protection des salariés (Falcoz, 2016).

En fait, à l'époque, je ne suis pas salarié que pour eux, il y a une période où je travaille dans quatre clubs différents, mais eux prennent le rôle de prestataires. C'est-à-dire que comme ils ne peuvent pas me payer entièrement, il y a une mutualisation. Les autres clubs payent une prestation à mon club et mon club me paye mon salaire. (S.3, homme, 37 ans)

\footnotetext{
${ }^{23}$ S.19 (homme, 40 ans) est éducateur en tir à l'arc et a été embauché par le groupement de son département. Il est ainsi mis à disposition de plusieurs communes de manière relativement stabilisée (avec lissage annuel : en moyenne $45 \mathrm{~h} /$ semaine, sur 36 semaines). Sa situation reste insatisfaisante : variation de temps de travail selon les années et salaire insuffisant pour lui (entre 1300 et 1700 euros).
} 
Cet entraîneur de tennis de table, qui ne se définit pas comme précaire mais n'est pas satisfait, semble être, comme les cas déjà évoqués, dans une situation d'entre-deux. Il est relativement stable, mais son emploi reste vulnérable, soumis à la capacité à maintenir les clubs-clients : « Chaque année pour moi ça bouge, je gagne un club, j'en perds un autre ». La possibilité d'avoir une situation stable dépend aussi des marchés spécifiques aux activités sportives. Dans les témoignages, l'accès à un ETP, y compris par de multiples employeurs, apparaît par exemple plus facile dans le tennis, l'escrime, la remise en forme (gymnastique volontaire, fitness) que dans les sports collectifs. Les maitres-nageurs accèdent également facilement à un temps de travail important entre club et collectivité sur un bassin.

Nombre d'interviewés reconnaissent vivre, ou avoir vécu, des situations de précarité professionnelle ${ }^{24}$. Pour autant ils témoignent assez régulièrement d'une réelle satisfaction du travail, y compris dans la multiplicité des petits emplois et une forme de déroutinisation de la vie professionnelle. Dans un domaine dominé par la passion et la compétition, on retrouve cette forme d'investissement vocationnel fait de disponibilité, de dévouement au club et de désintéressement (Chimot \& Schotté, 2006 ; Marsault et al., 2016). A cet égard, la branche sport semble se distinguer du secteur des services à la personne où l'on rentre par défaut et souvent sans qualification (Angeloff, 2008), ce qui pourrait favoriser un vécu et des stratégies d'employabilité rendant acceptable l'emploi à temps partiel. Cette ambivalence se retrouve-t-elle dans les dynamiques de précarité que l'on peut identifier dans les trajectoires professionnelles de ces salariés en quête de temps de travail?

\section{Dynamique de précarité dans les parcours professionnels}

Au sein des entretiens réalisés, trois types de trajectoires de précarités (en relation avec le temps partiel) ont pu être identifiés ${ }^{25}$ : les trajectoires de précarité initiale, envisagée

\footnotetext{
${ }^{24} \mathrm{Ce}$ constat est corroboré par une enquête quantitative (Garef-Sport, 2016) selon laquelle près d'une personne sur deux ayant un emploi principal dans la branche sport estime avoir connu précédemment des périodes de précarité.

${ }^{25}$ Les trajectoires et transitions professionnelles (Elder, 1985) sont ici entendues comme des séquences d'emploi inter-reliées et interdépendantes dont les dynamiques sont reconstruites a posteriori. À partir de la caractérisation initiale de la situation professionnelle (en termes de degré de stabilité et satisfaction, en comparant indicateurs objectifs de précarité et interprétation subjective par les interviewés) à l'entrée dans
} 
comme préalable «banalisé » à la stabilisation dans l'emploi ; les trajectoires rompues et déstabilisées alors même qu'elles semblaient s'être stabilisées; les trajectoires discontinues sans perspective (proche) de stabilisation. Ces trois formes d'expérience, inscrivant la précarité dans des temporalités différentes, rappellent, qu'à l'échelle des personnes, la précarité doit être appréhendée dans sa dimension «dynamique » (NicoleDrancourt, 1992) selon qu'elle renvoie à un parcours d'intégration ou d'exclusion.

\section{La précarité comme préalable à une stabilisation relative}

Ces trajectoires concernent des personnes qui, avant de se stabiliser dans un emploi, ont vécu des phases plus ou moins longues de précarité dans la branche sport (allant de 4 à 20 ans dans notre population). La majorité des salariés interviewés (32 personnes) ont vécu cette dynamique, alternance de (petits) emplois précaires et périodes de chômage, avec une difficulté à obtenir un ETP et un salaire satisfaisant. Cette "précarité d'intégration » (Nicole-Dancourt, 1992, p. 66) renvoie à des stratégies d'adaptation « laborieuse » (Paugam, 2000) pour lever les barrières d'accès à un emploi stable.

Ainsi par exemple, S.15 (femme de 28 ans) a un CQP Aide Monitrice de Voile qui lui a permis d'avoir un premier CDD de 8 mois dans une école de voile. Elle a ensuite passé son Brevet Professionnel de la Jeunesse, de l'Éducation Populaire et du Sport (BPJEPS) avec un contrat de professionnalisation. Puis elle a été au chômage et obtenu un CDD dans une base de loisir avant d'être embauchée comme monitrice de voile dans un club de sport nautique et de plaisance en tant que saisonnière en CDII. Son salaire est néanmoins faible et lissé sur 12 mois pour compenser les phases d'inactivité. Nous trouvons également des personnes cumulant les emplois dans plusieurs clubs/associations et finissant par avoir un CDI dans un des clubs (avec néanmoins souvent de faibles quotités horaires). En situation de multi-emplois, la pérennisation d'un seul des postes sur une quotité insuffisante créé une zone «d'entre-deux» (Bresson, 2011) dans la sécurisation de la situation professionnelle.

\footnotetext{
la branche, nous avons analysé les évolutions professionnelles ultérieures. Nous les avons regroupées par séquences en identifiant des événements marquants (ruptures biographiques ou transitions professionnelles plus progressives comme la «CDIsation » d'un même poste) en fonction de la logique à l'œuvre en termes d'intégration ou d'exclusion (ex. : continuité dans l'emploi ou augmentation du nombre d'employeurs sont des indicateurs d'un parcours d'intégration). Une telle analyse rétrospective oblige évidemment à une simplification et les dynamiques ne sont pas exclusives : un même salarié peut avoir vécu une précarité d'exclusion puis une précarité d'intégration par exemple.
} 
Dans notre échantillon, le temps pour obtenir un CDI satisfaisant (notamment en termes de quotité) varie majoritairement de 4 à 8 ans. Les premières formes de travail sont souvent des défraiements puis des CDD, la plupart du temps en contrat aidé ou en contrat de professionnalisation. D'autres ont accumulé des emplois de toute nature, dans et hors de la branche sport, avant de trouver un CDI avec un ETP (4 personnes sont dans ce cas de figure). Ces parcours évoquent les trajectoires peu voire non linéaires de sécurisation de l'insertion professionnelle des jeunes actifs (type 2 ou 3) décrites par Eckert et Mora (2008, p. 35sq.) : soit une discontinuité de la présence en emploi avec alternance de période de chômage, soit une installation durable en emploi sous statut temporaire, avant de réussir à pérenniser une situation professionnelle.

La stabilisation dans un emploi n'est cependant pas toujours un gage de satisfaction comme en témoigne S.8. Ce jeune homme de 26 ans a suivi un Master STAPS tout en travaillant comme éducateur sportif itinérant pour un comité départemental. Il quitte ce poste « stable » $(\mathrm{CDI})$ à temps très partiel (10h/semaine) et la branche du sport car il a une opportunité de s'engager dans une thèse financée avec une allocation et des enseignements à l'université. Il met fin à ce CDD de 3 ans à la fin de la première année. Pendant deux ans, il cumule les petits contrats en dehors de la branche (tourisme, habillement). En 2014, le comité départemental du sport en milieu rural lui propose de reprendre un CDI à temps plein. En 2016, il monte en responsabilité pour devenir conseiller technique départemental, mais avec une situation professionnelle dégradée (pas d'augmentation de salaire, accroissement de la charge de travail, gestion difficile des bénévoles). Il juge le niveau de rémunération très insatisfaisant à niveau Master et envisage au moment de l'entretien une réorientation professionnelle sans pour autant avoir de véritable projet professionnel alternatif.

La stabilité de l'emploi n'empêche pas une précarité du travail et un sentiment de dévalorisation. Précarité du travail et de l'emploi ne se superposent pas toujours, troublant la lecture univoque que l'on pourrait faire du secteur sportif.

\section{Ruptures et fragilités après stabilisation}

Pour les éducateurs sportifs de notre population, la stabilisation dans un emploi pérenne n'apparaît pas forcément comme un état durable : 33 interviewés témoignent dans leur 
parcours d'au moins une phase de fragilisation. L'obtention d'un CDI n'est pas toujours un gage d'assurance contre l'incertitude. Ce constat classique est accentué dans un secteur sportif caractérisé par la vulnérabilité des employeurs ou des situations d'emploi plus facilement fragilisées en cas de ruptures biographiques. On se retrouve ainsi dans une trajectoire renvoyant, au moins temporairement (si elle ne reste qu'une étape transitoire, l'éducateur trouvant les ressources pour une nouvelle intégration laborieuse) à une «précarité d'exclusion» (Nicole-Drancourt, 1992) du fait d'une exposition à des contraintes structurelles et/ou personnelles.

À l'image du secteur socio-culturel (Lebon, 2006), il existe ainsi une forte précarité non liée à la vulnérabilité du contrat mais à celle de l'emploi (Ghesquière, 2014), déstabilisé par la fragilité des employeurs qui restent encore souvent des associations sportives peu professionnalisées (Bernardeau-Moreau, 2004). Le manque de professionnalisation des structures et les carences des dirigeants sportifs en termes d'employeurabilité (Devetter \& Fleuriel, 2016) impacte dès lors fortement la qualité des emplois : insuffisance du management (manque de compétences, indifférence au développement), influence politique trop marquée, dépendance vis-à-vis des subventions ${ }^{26}$, manque de vision empêchant de pérenniser des emplois aidés, etc. (Falcoz \& Walter, 2007). On trouve ces mêmes problèmes d'employeurabilité dans d'autres secteurs et notamment dans ceux de l'aide à la personne dans lesquels les particuliers deviennent employeurs. L'enquête du CREDOC a ainsi montré que la grande majorité d'entre eux ne connaissent du cadre légal qui régit la relation à leur salarié que ce que contiennent les quelques informations données par le CESU (Chèque emploi service universel), et qu'ils n'ont pas d'outils pour gérer le recrutement des personnes ni les cas de conflit (Angotti, Brezault \& Loones, 2008). Dans les cas que nous avons rencontrés, les dirigeants de petites associations ont rarement de compétences en lien avec le métier d'employeur et leur choix de devenir président repose souvent sur leur engagement bénévole.

Ainsi, être en CDI n'est pas nécessairement gage de sécurité dans le secteur sportif. S.42 (homme de 42 ans) est entraîneur de taekwondo en CDI dans un club. Pourtant, il ne se juge pas stabilisé et protégé du fait de la mauvaise gestion de son employeur :

\footnotetext{
26 Dans le secteur associatif sportif, 30\% environ des ressources proviennent des subventions (contre $30 \%$ des cotisations de adhérents et encore environ 30\% de recette d'activité) (Beretti \& Calatayud, 2006).
} 
En ce moment mon poste est à deux doigts de sauter parce que le club n'a plus de sous, le président a joué avec la caisse depuis trois ans. Il a voulu embaucher un deuxième salarié et il l'a payé avec l'argent du Plan Sport Emploi qui m'avait été réservé, qu'on avait mis de côté, on avait 40000 euros dans les caisses.

Dans notre population, 5 éducateurs identifient les carences de management comme cause d'une précarisation professionnelle (licenciement, pertes d'heures). L'aléa sportif pèse aussi sur la stabilisation de l'emploi dans les clubs les plus ancrés dans le modèle compétitif ${ }^{27}$.

Le secteur tire parti de divers types de contrats où l'employeur bénéficie d'aides (exonération des cotisations sociales, subvention à l'embauche...) de l'État ou d'une collectivité (17\% des contrats sont aidés, Cereq, 2018). En abaissant les coûts liés à l'embauche, il s'agit de favoriser l'emploi de publics cibles tout autant que de pousser à limiter le travail informel et à faciliter le développement des structures employeuses (en particulier dans le secteur associatif). 7 interviewés ont été concernés par ces dispositifs (contrat d'avenir, emploi jeune, Plan Sport Emploi - dit « contrats aidés » ou contrats d'accompagnement pour l'emploi). À l'image de ce qui se passe dans les services à la personne (Angeloff, 2008) ou le secteur de l'animation (Lebon, 2013), ces dispositifs institutionnels restent des contrats instables. Inscrits dans un temps court, ils peuvent momentanément servir un parcours d'intégration pour des bénéficiaires déjà précarisés. Mais en dehors de quelques accès à des formations, dans un seul cas (S.44, avec une aide du Centre National pour le Développement du Sport - CNDS), l'aide a eu un effet levier, cofinançant l'emploi le temps de générer progressivement les conditions de l'autofinancement d'un poste pérenne et satisfaisant. Dans les autres situations, comme le suggère par ailleurs Falcoz (2016), l'emploi aidé tend à apparaître comme un effet d'aubaine permettant des recrutements opportunistes sans perspective de stabilisation. S.42, aujourd'hui entraineur salarié de Taekwondo, le constate de manière lapidaire :

Les clubs, ils prennent des contrats aidés, puis ils virent les mecs une fois que c'est terminé, et ils prennent un autre contrat aidé. Ça c'est tous les trois ans... Au karaté c'est pareil.

\footnotetext{
${ }^{27}$ Un Conseiller Technique Régional (CTR) de Handball (E.13) explique par exemple : « Mais prendre un $\mathrm{CDI}$, tu te dis je vais le prendre pour 20 ans sur une équipe sénior, il y a un peu plus de risques et c'est aussi pour ça qu'ils ne prennent pas de contrat. Il y a un turn-over qui est beaucoup plus grand chez les séniors que chez les enfants ».
} 
À une autre échelle, plus individuelle, les accidents biographiques sont identifiés comme un facteur de fragilisation par 18 interviewés. La naissance des enfants est régulièrement citée : il devient par exemple plus difficile (ou plus souhaité) de travailler sur les créneaux de fin de journée ou en logique saisonnière, ce qui fragilise le portefeuille d'activités. Les enjeux de revenus deviennent aussi plus pressants. La mobilité résidentielle (qui peut être liée à une rencontre) peut également remettre à plat tout le travail laborieux de constitution d'un réseau professionnel : il va falloir recréer dans un nouveau territoire un capital portefeuille d'employeurs. Une blessure, une maladie ou une séparation sont autant d'accidents biographiques (parfois nés de la précarisation) qui précipitent encore plus les difficultés. Si de tels facteurs sont communs à tous les secteurs professionnels, ils prennent une acuité plus grande du fait de certaines propriétés des métiers d'éducateur ${ }^{28}$ : engagement physique et maintien de son capital corporel, rôle marqué des réseaux dans les recrutements, horaires de travail atypiques...

Ainsi S.10 (femme, 34 ans), titulaire d'un BE, a vécu deux ruptures professionnelles liées à des bouleversements biographiques : un déménagement (qui fait perdre le réseau d'employeurs), puis plus tard une grossesse («je suis tombée enceinte, j'ai eu des difficultés avec l'employeur, du coup je me suis tournée vers autre chose »). À chaque fois, il a fallu relancer une dynamique de stabilisation, avec des détours temporaires en dehors de la branche (par exemple prendre un emploi alimentaire à temps partiel de vendeuse chez Décathlon). S. 39 (homme, 52 ans, BE tennis) a eu plusieurs bifurcations professionnelles du fait de facteurs personnels (déménagement, naissance de trois enfants) et un contexte général aujourd'hui peu propice au maintien du développement de ses activités d'entraîneur et de coach :

Il y a un déclin du tennis en général, il y a beaucoup moins de licenciés, beaucoup moins de personnes qui prennent des cours. Il y a la crise qui fait que les gens ont aussi moins d'argent pour s'inscrire dans les clubs pour prendre des cours... Pareil pour le coaching à domicile, et ça touche des gens assez favorisés, et du coup pour travailler que de ça, c'est difficile.

On perçoit ici combien l'emploi reste fragile au sens où l'éducateur sportif est soumis à des aléas et qu'il dispose de très peu de moyens pour y faire face (De Peretti, 2005). L'instabilité peut alors générer une inquiétude et un fort sentiment de vulnérabilité

\footnotetext{
${ }^{28}$ Des effets similaires ont été décrits pour le cas des animateurs socio-culturels (Lebon, 2010).
} 
(Paugam, 2000) mais qui reste d'une certaine manière pensé comme « normal » dans les règles du secteur.

\section{Des trajectoires sans stabilisation dans le secteur sportif}

Un autre groupe de personnes parmi nos interviewés (11) n'a jamais été et n'est pas encore stabilisé dans l'emploi : il se caractérise par des contrats précaires ou un nombre d'heures réalisées faible (8 sont en multi-emplois). Certains cas plutôt jeunes peuvent être interprétés comme étant dans une stratégie d'intégration juvénile qui n'aurait pas (encore ?) aboutie, mais une dynamique d'exclusion peut être précoce. S. 41 (homme, 28 ans, entraîneur rugby) est au chômage. Après un « rêve » avorté de joueur professionnel de rugby, il suit une formation privée de coach sportif mais n'arrive pas à se faire embaucher dans la salle de sport où il est stagiaire. Deux amis lancent une entreprise de coaching («qui marche très bien aujourd'hui ») à laquelle il essaye de s'associer (ils lui imposent un statut d'indépendant pour commencer).

Eux c'étaient des DEUST $^{29}$, ils avaient un diplôme universitaire que je n'avais pas moi. À l'époque les DEUST vampirisaient un peu le marché. En gros on avait les mêmes qualifications sauf qu'eux, ils étaient diplômés en université et moi dans une école privée... Moi ça m'a bloqué. (...) Après moi j'étais super jeune, eux un peu plus âgés et avec les clients grosso modo il fallait aller chez les clients, avec un sac, et faire du domicile, du coaching et ça n'a pas trop pris avec les clients parce que j'étais un peu juvénile du visage.

Sur la base d'une fragilité initiale (carrière de joueur pro avortée et absence de formation initiale), les barrières et les manques de capitaux (en particulier symbolique : reconnaissance de diplôme, expérience) le font arrêter au bout de huit mois et passer un BPJEPS pour essayer de travailler dans le rugby. Il se fait notamment recruter dans plusieurs mairies sur des emplois à très faibles volumes horaires (sous des statuts variés : pour un seul micro-CDI, des CDD voire du travail au noir à renouveler régulièrement). Il vit un sentiment d'éparpillement et de désillusion qui l'empêche de persévérer. Aujourd'hui au chômage depuis presque deux ans, il essaye de s'insérer dans une autre branche en cherchant une nouvelle formation.

\footnotetext{
${ }^{29}$ Diplôme d'études universitaires scientifiques et techniques.
} 
Mais plusieurs personnes ayant des profils plus âgés et des «carrières » déjà longues, interpellent, comme ce travailleur saisonnier (S.43, homme, 43 ans) : avec un CDD dans un club d'escalade, il complète son salaire en étant surveillant d'un parc accrobranches ou en ramassant des champignons. S.17 (homme de 40 ans) a également été joueur de rugby (période durant laquelle il dit avoir bien gagné sa vie). Il a ensuite passé des diplômes (notamment le Diplôme d'entraîneur), a été conseiller territorial sportif en CDI avec un salaire bas et est maintenant en CDII dans l'association support d'un club professionnel à seulement 25h/semaine.

Comme nous l'avons vu précédemment, cette forme de précarité est parfois évaluée comme inhérente au métier mais préférable à un emploi complètement stable. S.35 (femme de 44 ans) a par exemple été cadre de santé. Après avoir passé le BPJEPS (brevet professionnel de la jeunesse de l'éducation populaire et du sport), elle se consacre exclusivement à son travail dans le sport en multipliant les structures employeuses et en développant une approche sport-santé (club de basket, relais sport-santé, clubs de gymnastique volontaire) en tant que salariée mais aussi auto-entrepreneuse. Elle perçoit son travail comme précaire mais préfère cette situation à son CDI de cadre de santé :

C'est vrai que par rapport à l'hôpital, il y a beaucoup de précarité. C'est évident... Les contrats peuvent s'arrêter du jour au lendemain... L'hôpital ça reste moins précaire qu'une association. On sait bien une association du jour au lendemain, il suffit qu'il n'y ait plus de président. Elle peut se dissoudre. Et puis ce qui est compliqué quand on est éducatrice c'est que c'est difficile de faire son planning c'est-à-dire on va vous proposer les soirs, toujours aux mêmes heures, mais il n'y a que cinq soirs dans la semaine. Des horaires en journée, ce n'est pas évident d'en trouver. Donc pour se faire un planning à 35 heures dans ce métier, c'est quand même dur.

Un tel choix (emplois atypiques, perte de 1000 euros de salaire mensuel, réorganisation de la vie familiale) a été possible grâce à la stabilité de la situation professionnelle de son mari (dirigeant salarié d'un club de basket pérenne).

Un des enjeux des trajectoires est celui des perspectives professionnelles. L'acceptation durant un temps (il)limité d'une instabilité ou d'une incertitude vécue comme une norme au sein de la branche sport, amène à envisager la stabilisation par un changement de secteur. S.25 (homme, 23 ans) est entraineur de handball, salarié en tant qu'agent de développement au comité départemental et vacataire sur des missions de sélections. Il 
résume très bien cette ambivalence d'un secteur offrant beaucoup d'opportunités pour qui en accepte les incertitudes inhérentes.

Dans le handball c'est un peu compliqué pour entrainer à haut niveau, pour avoir des postes à haut niveau. Donc du coup je suis pas sûr de mon coup si je reste dans le handball. Je peux réussir comme je peux me casser la figure totalement. Je pourrais totalement quitter le handball, intégrer une mairie pour faire éducateur sportif, ou coach sportif en salle de musculation, avec les deux diplômes que j'ai, je pourrais faire ça. Après c'est pareil, c'est quand même assez précaire parce que c'est des contrats de 10/15h donc il faut les cumuler et c'est pas forcément des CDI, donc c'est à voir. Après, il y a quand même pas mal de boulot avec ça, donc il n'y a pas de soucis. Mais après, si je voulais vraiment avoir une situation stable, faudrait que je sorte du sport et me réorienter autre part, peut-être dans la finance ou des choses comme ça.

Comme dans le domaine socio-culturel « la progression de carrière suppose persévérance et capacité à se saisir d' « opportunités » en « voyant le vent venir », autant de contraintes et d'incertitudes qui conduisent certains vers d'autres métiers » (Lebon, 2013, p. 64). S.24 (femme de 51 ans) en est un exemple. Titulaire d'un BE, vacataire dans une mairie, entraineur dans un club et intervenante dans une association multisports, elle a été titularisée au bout de 20 ans dans la mairie pour laquelle elle travaillait comme contractuelle et a réduit ses heures dans son association. Plusieurs interviewés (4), après avoir multiplié les petits contrats, ont ainsi cherché un emploi dans un secteur plus stable en dehors de la branche sport, notamment via une titularisation tardive dans la fonction publique territoriale.

En définitive, ces entrées ou sorties tardives de la branche malgré la précarité, ou plus largement les alternances relativement acceptées de séquences d'intégration et d'exclusion, témoignent ainsi de cette figure oxymorique de l'« intégration incertaine » (Paugam, 2000) combinant rapport à l'emploi et perspective dégradés (insuffisance et instabilité des contrats) et rapport relativement satisfaisant au travail.

\section{Conclusion}

Les trajectoires professionnelles d'éducateurs sportifs en temps partiel mettent en évidence des logiques contrastées de recherche ou de soumission au travail à faible quotité, ainsi que de possibles dynamiques de précarisation ambivalentes. Certains travailleurs en emploi secondaire dans la branche sport choisissent d'avoir une activité 
subsidiaire (gratifiante et/ou lucrative), en préservant une forme d'indépendance et stabilité par leur emploi principal. Mais pour beaucoup d'interviewés, la précarité professionnelle apparaît subie et vécue comme une insécurisation de parcours d'autant plus forte que le sous-emploi se cumule avec d'autres indicateurs de précarité de l'emploi et du travail. Si le rapport salarial semble intégré comme un rapport atypique, il n'est pas pour autant dépourvu d'attraits et de satisfaction. Une précarité d'intégration renvoie à un passage transitoire vers des situations professionnelles plus stabilisées (par cumul d'emploi ou stabilisation dans un poste), assez caractéristiques des parcours professionnels juvéniles (Bouffartigue, 2015). Comme dans le milieu de l'animation (Lebon, 2013), un grand nombre de trajectoires d'intégration laborieuses peuvent être considérées comme ascendantes et en relative stabilisation, y compris par des bifurcations choisies ou contingentes vers d'autres métiers. Au sein de la branche professionnelle du sport, la stabilité de l'emploi passe d'abord par le cumul (d'emplois et/ou de qualifications). Néanmoins, il est frappant de constater la récurrence de trajectoires plus incertaines, y compris chez des travailleurs adultes : la perte d'activité et le chômage restent un horizon ordinaire (notamment du fait de la vulnérabilité des employeurs), même après des stabilisations; de plus certains éducateurs sportifs relativement âgés n'ont jamais connu de stabilisation dans la branche. Si une précarité d'exclusion est toujours possible, ce qui caractérise les expériences correspond plutôt à cet «entredeux », entre centre et périphérie, « entre deux normes, entre deux mondes, entre deux parcours de vie, qui semble la clé de lecture la mieux à même de caractériser et dynamiser la réflexion sur la précarité comme incertitude »(Bresson, 2011). Comme l'a suggéré Vultur (2010), nous avons vu que des emplois atypiques peuvent proposer des expériences professionnelles plutôt satisfaisantes. Dans une perspective plus critique on peut cependant constater que la qualité de l'emploi (y compris du point de vue économique) reste insuffisante pour offrir des perspectives professionnelles durables.

La branche professionnelle du sport apparaît bien comme un secteur professionnel ambivalent. L'accroissement de l'emploi d'éducateur sportif ne rime pas avec pérennisation de postes à temps plein, mais il offre - ou en donne l'impression - des opportunités pour un travailleur persévérant et passionné acceptant des formes variables de flexibilité. Moins concurrentiel et plus marqué par des carrières vocationnelles que le secteur des services à la personne où l'on arrive faute de mieux (Angeloff, 2008, Devetter 
\& Jany-Catrice, 2010), il partage avec celui-ci une structuration récente (par le haut) et une convention collective imparfaitement appliquée (Fleuriel, 2013, Devetter \& Fleuriel, 2016). Il semble à cet égard plus proche du secteur socio-culturel dont il partage beaucoup de caractéristiques, avec la spécificité d'une réglementation par les diplômes plus influente. Protection ou garantie de travail autant que barrière à l'entrée ou à l'évolution des carrières, les formations et les qualifications sont aussi un marché lucratif avec ses «zones grises »(Chevalier \& Pégard, 2016). Les éducateurs les plus « employables » sont ceux qui développent une polyvalence leur permettant de s'assurer un multi-emplois présentant la forme stable la plus courante dans la branche. Compte tenu de l'émiettement des situations professionnelles et des carences en termes d'« employeurabilité » des dirigeants sportifs, l'accès aux formations reste toutefois complexe, en particulier parce que méconnu (des salariés comme des employeurs) et surtout souvent onéreuses. Dans un contexte politique et économique mouvant (développement de nouveaux besoins sociaux, poursuite des baisses de financements publics, bouleversement de la formation tout au long de la vie, promotion de l'entrepreneuriat et « ubérisation » du travail ${ }^{30}$ ), dans quelle direction va s'orienter la création d'activité professionnelle dans le secteur sportif ? Va-t-on voir émerger une rationalisation - au sens sociologique - de la professionnalisation favorable à la qualité de l'emploi ? Ou bien l'individualisation de la relation salariale va-t-elle s'accentuer dans un marché atomisé d'éducateurs mués en entrepreneurs d'eux-mêmes ? L'avenir des éducateurs sportifs devra assurément être observé au regard de la montée en puissance des « zones grises d'emploi » (Azaïs et al., 2017) afin de décentrer l'analyse des seules relations employeurs-employés pour prendre en compte le rôle des autres parties prenantes (firmes-plateformes, fédérations sportives, État...).

\section{Références bibliographiques}

Abdelnour S. (2017), Moi, petite entreprise. Les auto-entrepreneurs, de l'utopie à la réalité, Paris : Presses universitaires de France.

30 L'ubérisation, au sens cette fois-ci de plateformisation numérique des relations (Vétois \& Raimbault, 2017), est une réalité en construction actuellement dans le secteur des éducateurs sportifs. Indice de l'émergence d'un « capitalisme de plateforme » (Abdelnour \& Bernard, 2018) dans le secteur sportif, des sociétés telles que « Coach 2 Store » se développent et proposent aux coachs et éducateurs sportifs un outil leur permettant de recommander des produits à leurs clients contre commission en cas d'achat. 
Abdelnour S. \& Bernard S. (2018) «Vers un capitalisme de plateforme ? Mobiliser le travail, contourner les régulations », La nouvelle revue du travail, vol. 13 [En ligne] http://journals.openedition.org/nrt/3797

Angeloff T. (2008), «Emploi de service: nouvelle domesticité ou gisement d'emplois ? », Idées économiques et sociales, vol. 153, n³, pp. 20-27.

Angotti M., Brezault M. \& Loones A. (2008), Étude sur le statut de particulier employeur, Paris, Rapport du CREDOC pour la FEPEM.

Azaïs C., Dieuaide P. \& Kesselman D. (2017), «Zone grise d'emploi, pouvoir de l'employeur et espace public : une illustration à partir du cas Uber », Relations industrielles, vol. 72, $\mathrm{n}^{\circ} 3$, pp. 433-456.

Barbier J.-C. (2005), «La précarité, une catégorie française à l'épreuve de la comparaison internationale », Revue française de sociologie, vol. 46, n², pp. 351-371.

Beretti E. \& Calatayud P. (2006), « Le budget des associations sportives ». Stat-infoBulletin de statistique et d'études, vol. 6, n ${ }^{\circ}$.

Bernardeau-Moreau D. (2004), Sociologie des fédérations sportives. La professionnalisation des dirigeants bénévoles, Paris, L'Harmattan.

Bernardeau-Moreau D. \& Collinet C. (dir.) (2009), Les éducateurs sportifs en France depuis 1945 : questions sur la professionnalisation, Rennes, Presses universitaires de Rennes.

Botsman R. \& Rogers R. (2011), What's Mine Is Yours: The Rise of Collaborative Consumption, Londres : Harper Collins Business.

Bouffartigue P. (2009), « Attendre le CDI pour faire grève et se syndiquer ? », in Béroud $\mathrm{S}$. et Bouffartigue P. (dir.), Quand le travail se précarise, quelles résistances collectives?, Paris, La Dispute, pp. 133-147.

Bouffartigue P. \& Béroud S. (dir.) (2009), Quand le travail se précarise, quelles résistances collectives?, Paris, La Dispute.

Bresson M. (2007), Sociologie de la précarité, Paris, Armand Colin.

Bresson M. (2011), « La précarité : une catégorie d'analyse pertinente des enjeux de la norme d'emploi et des situations sociales "d'entre-deux" », SociologieS [En ligne] http://journals.openedition.org/sociologies/3421

Camus J. (2008), «En faire son métier: de l'animation occasionnelle à l'animation professionnelle », Agora débats/jeunesses, vol. 2, pp. 32-44. 
Céreq (2018), Regards croisés sur le secteur des activités sportives et le métier d'éducateur sportif, Enquêtes, Portrait Statistique Emploi Formation, n².

Chevalier V. \& Pégard O. (2016), «L'emploi sportif : fabrique d'une illusion, fabrique à illusions », Marché et organisations, vol. 27, n³, pp. 15-29.

Chimot C. \& Schotté M. (2006), «Travailler dans une organisation sportive. Entre engagement passionné et investissement professionnel », Regards sociologiques, vol. 32, pp. 97-107.

Cingolani, P. (2005), La précarité, Paris, Presses universitaires de France.

De Peretti G. (2005), « Précaire : une catégorie instable et fragile », Empan, vol. 60, $\mathrm{n}^{\circ} 4$, pp. 14-23.

Deprost P., Laffon P. \& Imbaud D. (2013), Évaluation du régime de l'autoentrepreneur, Paris, Rapport IGF/IGAS.

Devetter F.-X. \& Jany-Catrice F. (2010), « L'invention d'un secteur et ses conséquences socio-économiques : les politiques de soutien aux services à la personne », Politiques et management public, vol. 27, $\mathrm{n}^{\circ}$ 2, [En ligne] http://journals.openedition.org/pmp/2256

Devetter F.-X. \& Fleuriel S. (2016), «Les relations professionnelles dans le sport : un processus inachevé », Marché et organisations, vol. 3, n²7, pp. 45-58.

Eckert H. (2010), "Précarité" dites-vous ?", SociologieS [En ligne] http://journals.openedition.org/sociologies/3285

Eckert H. \& Mora V. (2008), « Formes temporelles de l'incertitude et sécurisation des trajectoires dans l'insertion professionnelles des jeunes », Travail et Emploi, vol. 113, pp. $31-46$.

Elder G. (1985), «Perspective of the life course », in Elder G. (dir.), Life course dynamics, Ithaca, Cornell Université Press, pp. 23-49.

Falcoz M. (2013), «Entours et détours autour de l'emploi sportif», Socio-logos, vol. 8, [En ligne] https://socio-logos.revues.org/2742

Falcoz M. (2016), «Emplois sportifs, emplois pérennes, emplois précaires », Marché \& Organisations, vol. 27, pp. 27-48.

Falcoz M. \& Walter E. (2007), « Travailler dans un monde de bénévoles : contraintes et limites de la professionnalisation dans les clubs sportifs », RECMA, vol. 306, pp. 78-91. 
Falcoz M. \& Walter E. (2009), «L'emploi dans le sport associatif et fédéral, Un état de la question », STAPS, vol. 83, pp. 43-54.

Fleuriel S. (2013) « Ce que dialoguer veut dire. L'émergence du "dialogue social” dans le sport français (1990-2006) », Genèses, vol. 92, pp. 127-146.

Fleuriel S. (2016) «L'autre marché du travail et de l'emploi sportifs », Marché et organisations, vol. $3, \mathrm{n}^{\circ} 27$, pp. 11-14.

Fournier G., Bourassa B. \& Béli K. (dir.) (2003), La précarité au travail. Une réalité aux multiples visages, Laval, Les presses universitaires de Laval.

Garef-sport (2014), L'emploi sportif dans les régions, Rapport d'étude, CNPEF-sport.

Garef-sport (2016), Acquérir des compétences pour construire sa trajectoire professionnelle. Le défi de l'emploi à temps partiel dans la branche sport, Rapport d'étude, CNPEF-sport.

Ghesquière, F. (2014), «Précarité du contrat de travail et risque de perte d'emploi en Europe », Sociologie, vol. 5, n³, pp. 271-290.

Giraud O., Wolfarth M. T., Rey F. \& Lerrer Rosenfield C. (2014), «Les normes d'emploi au défi de l'auto-entreprenariat et des micro-entreprises individuelles. Une comparaison France Brésil », Revue Tiers Monde, vol. 218, pp. 35-52.

Gouju J.-L. (2014), «Emploi sportif : attention aux mirages », Les acteurs du sport, vol. 159 , p. 26.

Hely M. (2008), « À travail égal, salaire inégal ; ce que travailler dans le secteur associatif veut dire », Sociétés contemporaines, vol. 69, pp. 27-51.

Le Roux N. (2006), "Évolution des connaissances et perspectives de recherche sur l'emploi et la professionnalisation dans le secteur du sport », in Bouchet P. \& Pigeassou C. (dir), Management du sport: actualités, développements et orientations pour la recherche, Montpellier, AFRAPS, pp. 113-141.

Le Roux N. (dir.) (2002), L'emploi sportif en France : situation et tendance d'évolution, AFRAPS, RUNOPES.

Le Roux N. \& Aguettant N. (2006), «L'emploi sportif en France et ses évolutions : quels états des lieux ?» in Augustin J.-P. (dir.), Vers les métiers de l'animation et du sport : la transition professionnelle, Paris, La Documentation Française, pp. 147-165.

Lebon F. (2010), Les animateurs socioculturels, Paris, La Découverte. 
Lebon F. (2013), « Les animateurs entre précarité, militantisme et gestion politique des quartiers », Pensée plurielle, vol. 32, n¹, pp. 61-71.

Lebon F. \& de Lescure E. (2006), «Des "nouvelles professions" entre précarité et exibilité : animateurs socio-culturels et formateurs d'adultes (1982-2002)», Regards Sociologiques, vol. 32, pp. 83-95.

Loirand G. (2006), « Les obstacles au développement et à la stabilisation de l'emploi sportif », in Augustin J.-P. (dir.), Vers les métiers de l'animation et du sport : la transition professionnelle, Paris, La Documentation Française, pp. 245-258.

Malenfant R., LaRue A. \& Vézina M. (2007), « Intermittent Work and Well-being: One Foot in the Door », Current Sociology, vol. 55, n 6 , pp. 814-835.

Malet J. \& Bazin C. (2014), «Le sport en France : repères, chiffres clés et paroles d'acteurs », Recherches \& Solidarités.

Marsault C., Pichot L. \& Pierre J. (2016), « Le temps de travail atypique des éducateurs sportifs : entre contrainte et ressource identitaire », Formation Emploi, vol. 134, pp. 89105.

Méda D. (2017), « L'avenir du travail et de l'emploi à l'heure du numérique », Café de la statistique, <hal-01629446>

Nicole-Drancourt C. (1992), «L'idée de précarité revisitée », Travail et emploi, vol. 52, pp. 57-70.

Nirello L. \& Prouteau L. (2016), « Des salariés associatifs moins bien payés ? », Travail et Emploi, vol. 148, [En ligne] http://travailemploi.revues.org/7361

Omnas (2006), Enquête auprès des entreprises de la branche sport, Paris.

Ortiz A. (2009), « Trajectoire professionnelle et état de santé déclaré des salariés seniors en activité », Revue économique, vol. 60, n², pp. 365-383.

Paugam S. (2000), Le salarié de la précarité, Paris, Presses Universitaires de France.

Pierre J. \& Collinet C. (2014), « De l'OMNAS au CAFEMAS. Naissance et disparition d'un dispositif au service des politiques de l'emploi sportif », Revue européenne de management du sport, vol. 4, pp. 18-31.

Quadrat-Étude (2018) Rapport de branche, http://www.cpnefsport.com/Documents/Quadrat-Pluricite_Sport_R\%20apport_de_branche_2018.pdf 
Ribault T. (2008), «Aide à domicile : de l'idéologie de la professionnalisation à la pluralité des professionnalités », Revue Française de Socio-Économie, Vol. 2, n², pp. 99-117.

Tasset C. (2017) «La mesure des précaires. Revisiter la genèse de l'idée de précarité à la lumière des rapports à la quantification », Sociologies et Société, vol. XLIX, n²1, pp. 215-237.

Ulrich V. (2009), « Logiques d'emplois à temps partiel et trajectoires professionnelles des femmes », in Ariane Pailhé et al. (dir.), Entre famille et travail, Paris, La Découverte, pp. 209-232.

Vétois P. \& Raimbault N. (2017), " L'“ubérisation" de la logistique : disruption ou continuité ? Le cas de l'Ile-de-France », Technologie et innovation, vol. 3. [En ligne] http://www.openscience.fr/L-uberisation-de-la-logistique-disruption-ou-continuite-Lecas-de-l-Ile-de

Villeneuve A. (1984), « Construire un indicateur de précarité : les étapes d'une démarche empirique », Économie et statistique, vol. $168, n^{\circ} 1$, pp. 93-105.

Vultur M. (2010), «La précarité : un “concept fantôme”" dans la réalité mouvante du monde du travail », Sociologies http://journals.openedition.org/sociologies/3287

Weber F. (2008), Le travail au noir : une fraude parfois vitale ?, Paris, Éditions Rue d'Ulm. 
Annexe 1 : Liste des salariés interrogés

\begin{tabular}{|c|c|c|c|}
\hline Numéro & Age & Sexe & Fonction actuelle principale \\
\hline 1 & 23 ans & Homme & $\begin{array}{l}\text { Éducateur PSG Academy et employé } \\
\text { polyvalent }\end{array}$ \\
\hline 2 & 37 ans & Homme & $\begin{array}{ll}\text { Intermittent } & \text { musicien/moniteur } \\
\text { d'escalade } & \end{array}$ \\
\hline 3 & 37 ans & Homme & Éducateur en tennis de table \\
\hline 4 & 45 ans & Homme & Éducateur de tennis \\
\hline 5 & 48 ans & Femme & Éducatrice de gymnastique volontaire \\
\hline 6 & 37 ans & Femme & Éducatrice en fitness et en danse \\
\hline 7 & 49 ans & Homme & $\begin{array}{lll}\text { Employé entreprise/éducateur en } \\
\text { Kung Fu }\end{array}$ \\
\hline 8 & 26 ans & Homme & $\begin{array}{l}\text { Éducateur en tennis de table et } \\
\text { conseiller technique au sein de la } \\
\text { fédération sportive du monde rural }\end{array}$ \\
\hline 9 & 50 ans & Homme & Professeur EPS/entraîneur de rugby \\
\hline 10 & 34 ans & Homme & Éducateur dans un club de tennis \\
\hline 11 & 26 ans & Femme & Éducatrice dans un club de tennis \\
\hline 12 & 26 ans & Femme & Éducatrice en voile \\
\hline 13 & 25 ans & Homme & Maitre-nageur sauveteur \\
\hline 14 & 54 ans & Homme & Éducateur en kayak \\
\hline 15 & 28 ans & Femme & Éducatrice en voile \\
\hline 16 & 28 ans & Homme & $\begin{array}{l}\text { Éducateur en activités physiques } \\
\text { adaptées }\end{array}$ \\
\hline 17 & 40 ans & Homme & $\begin{array}{l}\text { Éducateur en rugby et Responsable } \\
\text { centre de formation associatif en } \\
\text { rugby }\end{array}$ \\
\hline 18 & 22 ans & Homme & $\begin{array}{l}\text { Saisonnier éducateur sur parcours } \\
\text { d'accrobranche }\end{array}$ \\
\hline 19 & 39 ans & Homme & Éducateur en tir à l'arc \\
\hline 20 & 47 ans & Homme & Éducateur en handball \\
\hline 21 & 25 ans & Homme & $\begin{array}{l}\text { Vendeur Décathlon/préparateur } \\
\text { physique en football }\end{array}$ \\
\hline 22 & 31 ans & Homme & Maitre-nageur sauveteur \\
\hline 23 & 29 ans & Homme & $\begin{array}{l}\text { Agent bancaire/ Maitre-nageur } \\
\text { sauveteur }\end{array}$ \\
\hline 24 & 51 ans & Femme & $\begin{array}{ll}\text { Employée } & \text { mairie/éducatrice } \\
\text { polyvalente } & \\
\end{array}$ \\
\hline 25 & 23 ans & Homme & $\begin{array}{l}\text { Agent développement Comité } \\
\text { départemental/Entraîneur de handball }\end{array}$ \\
\hline 26 & 38 ans & Homme & Entraineur de tennis \\
\hline
\end{tabular}




\begin{tabular}{|c|c|c|c|}
\hline 27 & 53 ans & Homme & $\begin{array}{l}\text { Conseiller technique régional en } \\
\text { tennis/éducateur en tennis }\end{array}$ \\
\hline 28 & 46 ans & Homme & $\begin{array}{lll}\text { Directeur } & \text { sportif } & \text { club } \\
\text { tennis/entraineur de tennis } & \end{array}$ \\
\hline 29 & 30 ans & Homme & Éducateur en athlétisme \\
\hline 30 & 52 ans & Homme & Prof EPS ESPE/entraîneur de tennis \\
\hline 31 & 36 ans & Homme & Salarié mairie/éducateur polyvalent \\
\hline 32 & 20 ans & Homme & Étudiant/ Maitre-nageur sauveteur \\
\hline 33 & 20 ans & Homme & Étudiant/Maitre-nageur sauveteur \\
\hline 34 & 31 ans & Femme & Éducatrice en tennis \\
\hline 35 & 44 ans & Femme & Éducatrice polyvalente \\
\hline 36 & 35 ans & Homme & Entraineur de tennis \\
\hline 37 & 43 ans & Femme & $\begin{array}{l}\text { Professeur d'EPS/ Maitre-nageur } \\
\text { sauveteur }\end{array}$ \\
\hline 38 & 30 ans & Homme & $\begin{array}{l}\text { Professeur } \quad \text { de } \\
\text { militaire/éducateur en roller }\end{array}$ \\
\hline 39 & 29 ans & Homme & Éducateur en Sport santé \\
\hline 40 & 54 ans & Homme & Éducateur en Sport santé \\
\hline 41 & 28 ans & Homme & Entraineur rugby \\
\hline 42 & 42 ans & Homme & Entraineur de Taekwondo \\
\hline 43 & 43 ans & Homme & Saisonnier/éducateur en escalade \\
\hline 44 & 32 ans & Homme & $\begin{array}{l}\text { Salarié comité départemental } \\
\text { athlétisme/entraîneur d'athlétisme }\end{array}$ \\
\hline
\end{tabular}


Annexe 2 : Liste des employeurs interrogés

\begin{tabular}{|c|c|c|}
\hline $\begin{array}{l}\text { Numéro de } \\
\text { l'entretien }\end{array}$ & $\begin{array}{l}\text { Statut des personnes } \\
\text { interrogées }\end{array}$ & Nature de la structure \\
\hline 1 & Directeur technique & $\begin{array}{l}\text { Association liée à un club du top } 14 \\
\text { (rugby) }\end{array}$ \\
\hline 2 & Vice-président & $\begin{array}{l}\text { Ligue de Sport adapté et Comité } \\
\text { départemental de Sport en milieu } \\
\text { rural }\end{array}$ \\
\hline 3 & Président du club & $\begin{array}{l}\text { Club de gymnastique dépendant } \\
\text { d'une structure omnisport : I'UMS }\end{array}$ \\
\hline 4 & Président & Club de tennis \\
\hline 5 & Gérante & Salle privée d'arts martiaux \\
\hline 6 & Directeur de centre & Centre Urban Soccer \\
\hline 7 & Gérant & $\begin{array}{l}\text { Accro'parc. Base de loisir multi } \\
\text { activités }\end{array}$ \\
\hline 8 & Directeur général & Tremplin Sport Formation \\
\hline 9 & Référent national/ Expert VTT & UCPA \\
\hline 10 & Présidente & Club de judo \\
\hline 11 & Présidente & Club de Sport pour tous \\
\hline 12 & Gérant & Club de remise en forme \\
\hline 13 & $\begin{array}{l}\text { Conseillère technique } \\
\text { régionale }\end{array}$ & Ligue de hand-ball \\
\hline 14 & Président de fédération & Fédération d'art martial \\
\hline 15 & $\begin{array}{l}\text { Directeur administratif centre } \\
\text { de formation }\end{array}$ & Club professionnel de rugby \\
\hline 16 & $\begin{array}{l}\text { Directeur général adjoint club } \\
\text { professionnel }\end{array}$ & $\begin{array}{l}\text { Club de basket + centre de formation } \\
+ \text { association }\end{array}$ \\
\hline 17 & $\begin{array}{c}\text { Gestionnaire des salariés (+ } \\
\text { éducatrice) }\end{array}$ & Club d'escalade \\
\hline 18 & Président d'association & Association de Sport-santé \\
\hline 19 & Secrétaire générale & Sociétés de Sport- santé \\
\hline 20 & Directeur adjoint piscine & RECREA \\
\hline 21 & $\begin{array}{l}\text { Directeur technique et } \\
\text { financier }\end{array}$ & Paris University Club (PUC) \\
\hline 22 & Conseiller en développement & Ligue de tennis \\
\hline 23 & Responsable d'exploitation & RECREA \\
\hline 24 & DTN adjoint & Fédération de Hand-ball \\
\hline 25 & Superviseur & Urban Soccer Lognes \\
\hline 26 & Directeur & Base de loisir UCPA \\
\hline 27 & Président & Club de hand-ball \\
\hline 28 & Président & Profession sport \\
\hline
\end{tabular}




\begin{tabular}{|c|c|c|c|c|}
\hline 29 & $\begin{array}{l}\text { Co-gérant bureau des } \\
\text { moniteurs Escalade et } \\
\text { Canyoning }\end{array}$ & $\begin{array}{l}\text { Bureau des } \\
\text { Canyon }\end{array}$ & moniteurs & Escalade- \\
\hline
\end{tabular}

